\title{
Neutrophil-to-Lymphocyte Ratio and Platelet-to-Lymphocyte Ratio in Patients with Sudden Sensorineural Hearing Loss
}

\author{
Xiao-Feng Qiao ${ }^{a} \quad$ Xin Li ${ }^{b} \quad$ Guo-Ping Wang ${ }^{a} \quad Y^{\prime}$-Huan Bai ${ }^{a}$ Wei Zheng ${ }^{a}$ \\ Tong-Li Li ${ }^{\mathrm{a}}$ \\ aDepartment of Otorhinolaryngology, Shanxi Provincial People's Hospital Affiliated to Shanxi Medical University, \\ Taiyuan, China; ${ }^{b}$ Department of Surgery, Children's Hospital of Shanxi Province, Taiyuan, China
}

\section{Significance of the Study}

- This study shows that the peripheral blood neutrophil-to-lymphocyte ratio (NLR) and platelet-tolymphocyte ratio (PLR) are associated with the outcome and prognosis of sudden sensorineural hearing loss (SSNHL). A higher NLR in patients with SSNHL suggests local inflammation, while a higher PLR suggests pathological damage of endothelial cells. The detection of NLR and PLR can be used as the basis for individualized treatment of SSNHL as it is convenient, reliable, and economical.

\section{Keywords}

Sudden sensorineural hearing loss · Neutrophils ·

Lymphocytes · Platelets · Ratios

\begin{abstract}
Objective: Sudden sensorineural hearing loss (SSNHL) is a common acute disease with an incidence of 0.5-2/10,000. This study aimed to determine whether the neutrophil-tolymphocyte ratio (NLR) and the platelet-to-lymphocyte ratio (PLR) could be indicators for SSNHL. Methods: A total of 60 confirmed cases of SSNHL and 60 healthy volunteers were included in this study. Peripheral blood NLRs and PLRs were compared between these groups. The SSNHL patients were divided into two groups, according to therapeutic effect: an effective group and an ineffective group. Peripheral blood NLRs and PLRs before and after treatment were compared between these two groups. Results: The average NLRs and
\end{abstract}

\begin{tabular}{ll}
\hline KARGER & $\begin{array}{l}\text { (c) } 2018 \text { The Author(s) } \\
\text { Published by S. Karger AG, Basel }\end{array}$ \\
E-Mail karger@karger.com & $\begin{array}{l}\text { This is an Open Access article licensed under the Creative Commons } \\
\text { Attribution-NonCommercial-4.0 International License (CC BY-NC) } \\
\text { (http://www.karger.com/Services/OpenAccessicense), applicable to } \\
\text { the online version of the article only. Usage and distribution for com- } \\
\text { mercial purposes requires written permission. }\end{array}$
\end{tabular}

PLRs of these patients were both significantly higher than in controls. The average NLRs and PLRs of the ineffective group were both significantly higher than those of the effective group. Conclusion: Peripheral blood NLR and PLR could be used as a convenient, reliable, and cost-effective indicator to predict the prognosis of SSNHL. @ 2018 The Author(s)

Published by S. Karger AG, Basel

\section{Introduction}

Sudden sensorineural hearing loss (SSNHL) is a common acute disease with an incidence of $0.5-2 / 10,000$ [1, 2]. Many causes have been proposed; these include viral infection, vascular disturbance, microcirculatory failure, and immune mediation, but none of these are supported by definitive evidence [3-5]. Recent etiological research on SSNHL has focused on chronic inflammation [6]. Bac- 
teria- or virus-induced chronic inflammation may cause microvascular injuries and atherosclerosis, raising the risks of cochlear ischemia [7]. However, cochlear ischemic alteration is just one potential pathological change of SSNHL with a vascular cause. Chronic inflammation can also induce corresponding endocochlear immune responses, further leading to cochlear injuries [3]. Researchers in cardiovascular diseases have used the neutrophil-to-lymphocyte ratio (NLR) in peripheral blood as a marker for cardiovascular diseases, and it may serve as a useful predictor of development of cardiovascular disease. Furthermore, the platelet-to-lymphocyte ratio (PLR) has been closely correlated with peripheral arterial occlusion diseases such as arteriosclerosis and arterial thrombosis [8]. Hence, elevation of peripheral NLR and PLR indicates the occurrence of vascular endothelial injury, atherosclerosis, and local microartery inflammation. On this basis, we hypothesized that peripheral NLR and PLR can also be used to predict the metastasis/development of SSNHL and applied as markers to easily measure prognosis. In the present study, we aimed to investigate the relationship of SSNHL with NLR and PLR, in an attempt to validate our hypothesis.

\section{Materials and Methods}

\section{Clinical Data}

A total of 60 SSNHL patients treated at Shanxi Provincial People's Hospital between December 2015 and December 2016 (average age: $45.62 \pm 13.16$ years; male: 28 ; female: 32 ), according to the Sudden Hearing Loss Diagnosis and Treatment Guidelines of the Chinese Medical Association [9], were included in the study. In addition, 60 healthy subjects were assigned as the control group (average age: $49.62 \pm 10.66$ years; male: 31 ; female: 29 ). The inclusion criteria were as follows: no history of steroid treatment, blood abstraction and pure-tone hearing inspection before treatment, and magnetic resonance imaging excluding hearing loss due to organic lesions. Subjects with the following diseases were excluded: any acute inflammatory infection, diabetes, hypertension, hyperlipidemia, coronary diseases, acute or chronic kidney failure, chronic liver disease, lung disease, infectious diseases (e.g., syphilis), immunological diseases, and any otological diseases (e.g., chronic otitis, otosclerosis, history of ear trauma, and Meniere's disease).

\section{Methods}

Blood specimens were examined before the treatment. Based on medical history and test results, unqualified cases were excluded, while the included cases were assigned to the same therapeutic scheme.

\section{Peripheral Blood Laboratory Examination}

Peripheral blood was collected from all included subjects before treatment and 15 days after treatment, followed by routine blood cell analysis. The sample collection was done between 7 and
Table 1. Peripheral blood data in patients and controls

\begin{tabular}{lccc}
\hline Parameter & $\begin{array}{c}\text { Patients } \\
(n=60)\end{array}$ & $\begin{array}{l}\text { Controls } \\
(n=60)\end{array}$ & $\begin{array}{l}p \\
\text { value }\end{array}$ \\
\hline WBC count, $\times 10^{9} / \mathrm{L}$ & $8.38 \pm 1.33$ & $5.24 \pm 1.39$ & 0.001 \\
Neutrophil count, $\times 10^{9} / \mathrm{L}$ & $7.23 \pm 1.41$ & $3.57 \pm 1.02$ & 0.000 \\
Platelet count, $\times 10^{9} / \mathrm{L}$ & $263.90 \pm 47.90$ & $201.45 \pm 41.11$ & 0.356 \\
Lymphocyte count, $\times 10^{9} / \mathrm{L}$ & $2.20 \pm 1.00$ & $1.88 \pm 0.57$ & 0.022 \\
NLR & $3.96 \pm 1.92$ & $2.33 \pm 0.97$ & 0.000 \\
PLR & $145.69 \pm 74.66$ & $117.30 \pm 44.06$ & 0.003 \\
Age & $45.62 \pm 13.16$ & $49.62 \pm 10.66$ & 0.081 \\
& & &
\end{tabular}

NLR, neutrophil-to-lymphocyte ratio; PLR, platelet-to-lymphocyte ratio; WBC, white blood cell.

8 a.m. and the subjects were in a fasting state. The absolute counts of neutrophils, lymphocytes, and platelets in peripheral blood were obtained and the NLR and PLR of each case computed.

\section{Detection of Pure-Tone Hearing}

For the 60 patients, pure-tone hearing was measured before treatment and 15 days after treatment. Specifically, the bone conduction threshold and air conduction threshold at 250, 500, 1,000, $2,000,4,000$, and $8,000 \mathrm{~Hz}$ were recorded. According to the therapeutic effect evaluation standards of the Sudden Hearing Loss Diagnosis and Treatment Guidelines of the Chinese Medical Association, patients who achieved "cured," "markedly effective," and "effective" outcomes were classified as the "effective group," and patients who achieved "ineffective" outcome were classified as the "ineffective group."

\section{Treatment Regimen}

All 60 patients were treated with the following regimen: intravenous injection of $10 \mathrm{mg}$ of dexamethasone for 3 consecutive days and intravenous drip injection of $70 \mathrm{mg}$ of ginkgo biloba extract (Ginaton) for 15 consecutive days.

\section{Statistical Methods}

The average peripheral NLR and PLR were tested via $t$ test for the test group versus control group and the effective group drip injection ineffective group on SPSS 20. The NLR and pure-tone hearing thresholds were examined on the straight line correlation analysis.

\section{Results}

The white blood cell (WBC) count, neutrophil count, lymphocyte count, NLR, and PLR of SSNHL patients before the treatment were all significantly higher than those of the controls (Table 1).

The lymphocyte count and NLR were both significantly different before treatment in the effective group compared to the ineffective group $(p<0.05)$. After treatment, 
Table 2. Peripheral blood data in the effective and ineffective subgroups

\begin{tabular}{|c|c|c|c|c|c|c|}
\hline \multirow[t]{2}{*}{ Parameter } & \multicolumn{3}{|c|}{ Before treatment } & \multicolumn{3}{|c|}{ After treatment } \\
\hline & $\begin{array}{l}\text { effective } \\
(n=48)\end{array}$ & $\begin{array}{l}\text { ineffective } \\
(n=12)\end{array}$ & $\begin{array}{l}p \\
\text { value }\end{array}$ & $\begin{array}{l}\text { effective } \\
(n=48)\end{array}$ & $\begin{array}{l}\text { ineffective } \\
(n=12)\end{array}$ & $\begin{array}{l}p \\
\text { value }\end{array}$ \\
\hline WBC count, $\times 10^{9} / \mathrm{L}$ & $8.31 \pm 1.32$ & $8.65 \pm 1.42$ & 0.124 & $6.24 \pm 1.06$ & $8.74 \pm 1.20$ & 0.018 \\
\hline Neutrophil count, $\times 10^{9} / \mathrm{L}$ & $7.26 \pm 1.41$ & $7.10 \pm 1.48$ & 0.245 & $6.68 \pm 1.27$ & $7.16 \pm 0.99$ & 0.157 \\
\hline Platelet count, $\times 10^{9} / \mathrm{L}$ & $256.15 \pm 50.16$ & $226.08 \pm 54.08$ & 0.350 & $199 \pm 50.02$ & $212 \pm 34.87$ & 0.351 \\
\hline Lymphocyte count, $\times 10^{9} / \mathrm{L}$ & $2.62 \pm 1.22$ & $1.54 \pm 0.56$ & 0.002 & $2.91 \pm 1.59$ & $1.45 \pm 1.44$ & 0.285 \\
\hline NLR & $3.39 \pm 1.71$ & $5.21 \pm 2.27$ & 0.031 & $2.45 \pm 1.45$ & $5.03 \pm 1.68$ & 0.000 \\
\hline PLR & $132.81 \pm 60.89$ & $154.23 \pm 63.85$ & 0.258 & $121.02 \pm 46.98$ & $158.31 \pm 52.38$ & 0.025 \\
\hline
\end{tabular}

NLR, neutrophil-to-lymphocyte ratio; PLR, platelet-to-lymphocyte ratio; WBC, white blood cell.

Table 3. Correlation analysis between NLR and hearing thresholds before and after treatment in the SSNHL patients

\begin{tabular}{|c|c|c|c|c|c|c|}
\hline \multirow[t]{2}{*}{ Subgroup } & \multicolumn{3}{|c|}{ Before treatment } & \multicolumn{3}{|c|}{ After treatment } \\
\hline & NLR & hearing threshold & $r$ & NLR & hearing threshold & $r$ \\
\hline Effective & $3.39 \pm 1.71$ & $48.62 \pm 16.62$ & 0.45 & $2.45 \pm 1.45$ & $22.81 \pm 11.80$ & 0.57 \\
\hline Ineffective & $5.21 \pm 2.27$ & $60.42 \pm 11.77$ & 0.64 & $5.03 \pm 1.68$ & $57.50 \pm 13.06$ & 0.54 \\
\hline
\end{tabular}

NLR, neutrophil-to-lymphocyte ratio; SSNHL, sudden sensorineural hearing loss.

Table 4. Correlation analysis between NLR and age before and after treatment in the SSNHL patients

\begin{tabular}{|c|c|c|c|c|c|c|}
\hline \multirow[t]{2}{*}{ Subgroup } & \multicolumn{3}{|c|}{ Before treatment } & \multicolumn{3}{|c|}{ After treatment } \\
\hline & NLR & age & $r$ & NLR & age & $r$ \\
\hline Ineffective & $5.21 \pm 2.27$ & $45.10 \pm 11.29$ & 0.14 & $5.03 \pm 1.68$ & $45.10 \pm 11.29$ & 0.11 \\
\hline
\end{tabular}

NLR, neutrophil-to-lymphocyte ratio; SSNHL, sudden sensorineural hearing loss.

a significant difference in the effective group versus the ineffective group was found in WBC count, NLR, and PLR, but not in neutrophil count, platelet count, or lymphocyte count $(p>0.05)$ (Table 2).

The NLR was significantly and positively correlated with hearing thresholds both before and after treatment among SSNHL patients $(p<0.05)$ (Table 3$)$.

In the treatment group and the control group there was no correlation between NLR and age $(r=0.07$ and $r=0.13$, respectively). In the effective treatment group and the ineffective treatment group, there was no correlation between NLR and age before and after treatment, indicating that for patients, NLR in the peripheral blood was not influenced by age. Details are depicted in Table 4 .

Blood Cell Ratios in Patients with Sudden Sensorineural Hearing Loss

\section{Discussion}

SSNHL is a common acute disease in otolaryngology that mainly manifests as sudden acute deafness. SSNHL is defined as sudden hearing loss without definite cause within $72 \mathrm{~h}$, with a hearing drop of $\geq 20 \mathrm{~dB}$ in at least two adjacent frequencies [9]. However, the precise pathophysiological mechanisms underlying SSNHL remain unknown and may be induced by local and systemic factors. A number of hypotheses have explained the cause of SSNHL, such as immune reactions, microcirculatory disturbance, infection, infectious disease, and tumors. Many researchers have suggested viral etiologies, but antiviral treatment has not achieved a satisfactory effect. To date, 
there are no specific reports on serological characteristics. Merchant et al. [4] studied the temporal bone histopathology of 15 deaf ears and found viral particles without microscopy; thus, no direct cochlear evidence has been found for viral infection. No immune- or autoimmune-mediated injuries have been reported [10], indicating a lack of support for possible immune etiologies. The thrombosis hypothesis regarding the pathogenesis of SSNHL should be questioned because there was no difference in incidence rate between males and females [11]. Existing etiological research on SSNHL has focused on chronic inflammation. It has been believed that bacteria- or virus-induced chronic inflammation could cause microvascular injury, vascular endothelial dysfunction, and atherosclerosis, leading to an increased risk of cochlear ischemia [7, $12,13]$. Furthermore, susceptibility to genetic thrombosis or cardiovascular risk factors would further aggravate the risk of cochlear ischemia $[14,15]$. The NLR in peripheral blood has not only been proved to be one of the definitive markers of chronic inflammation in the cardiovascular system, it is also a reliable predictor of functional recovery after vascular disease. Furthermore, this index is easy to measure in clinical practice [16-18]. Moreover, the PLR is closely correlated with peripheral arterial occlusion diseases such as arteriosclerosis and arterial thrombosis [8]. Hence, the elevation of peripheral NLR and PLR indicates the occurrence of atherosclerosis and local microartery inflammation [19-23]. In the present study, we aimed to investigate the relationship of SSNHL with NLR and PLR.

The WBC count, neutrophil count, lymphocyte count, NLR, and PLR of SSNHL patients before treatment were all significantly higher than those of the controls. The lymphocyte count and NLR before treatment in the effective group were both significantly lower than those in the ineffective group. After treatment, WBC count, NLR, and PLR were all significantly different between groups, while NLR was positively correlated with hearing thresholds in SSNHL patients both before and after treatment. This indicates that the NLR can be considered for use as a predictor of SSNHL.

A higher peripheral NLR suggests the occurrence of local microartery vascular inflammation, which involves the labyrinthine artery. However, the NLR before treatment was higher in the ineffective group than in the effective group, indicating that the cochlear microvascular local inflammation was more severe. Thus, as for treatment, systemically or locally used glucocorticoid exerts an anti-inflammatory effect and reduces the microvascular local inflammatory reaction, which involves the labyrinthine artery [24]. This indicates the achievement of a satisfactory therapeutic effect [9]. A larger PLR indicates that a patient with SSNHL may be suffering from pathological injury to peripheral vascular endothelial cells that involves the labyrinthine artery. On this basis, antioxidant drugs and antiplatelet aggregative drugs may be beneficial. As for the ineffective group, the PLR after treatment increased relative to the effective group, suggesting that the more severe injuries to vascular endothelial cells and the formation of atherosclerotic plaques accounted for the bad prognosis in these ineffective patients, which should be taken into consideration for therapy.

\section{Conclusion}

The NLR can be considered for use as a marker for prognosis and the development of SSNHL, while the NLR and the PLR can be considered for use as a basis for personalized therapy.

\section{References}

1 Stachler RJ, Chandrasekhar SS, Archer SM, Rosenfeld RM, Schwartz SR, Barrs DM, et al.; American Academy of Otolaryngology-Head and Neck Surgery. Clinical practice guideline: sudden hearing loss. Otolaryngol Head Neck Surg. 2012 Mar; 146(3 Suppl):S1-35.

2 Schreiber BE, Agrup C, Haskard DO, Luxon LM. Sudden sensorineural hearing loss. Lancet. 2010 Apr;375(9721):1203-11.

3 Masuda M, Kanzaki S, Minami S, Kikuchi J, Kanzaki J, Sato H, et al. Correlations of inflammatory biomarkers with the onset and prognosis of idiopathic sudden sensorineural hearing loss. Otol Neurotol. 2012 Sep;33(7):1142-50.
4 Merchant SN, Durand ML, Adams JC. Sudden deafness: is it viral? ORL J Otorhinolaryngol Relat Spec. 2008;70(1):52-60.

5 Ryan AF, Harris JP, Keithley EM. Immunemediated hearing loss: basic mechanisms and options for therapy. Acta Otolaryngol Suppl. 2002;548(548):38-43.

6 Hiramatsu M, Teranishi M, Uchida Y, Nishio $\mathrm{N}$, Suzuki H, Kato K, et al. Polymorphisms in genes involved in inflammatory pathways in patients with sudden sensorineural hearing loss. J Neurogenet. 2012 Sep;26(3-4):387-96.

7 Hoffman M, Blum A, Baruch R, Kaplan E, Benjamin M. Leukocytes and coronary heart disease. Atherosclerosis. 2004 Jan;172(1):1-6.
8 Gary T, Pichler M, Belaj K, Hafner F, Gerger A, Froehlich H, et al. Platelet-to-lymphocyte ratio: a novel marker for critical limb ischemia in peripheral arterial occlusive disease patients. PLoS One. 2013 Jul;8(7):e67688.

9 Editorial Board of Chinese Journal of Otorhinolaryngology Head and Neck Surgery; Society of Otorhinolaryngology Head and Neck Surgery, Chinese Medical Association. [Guideline of diagnosis and treatment of sudden deafness (2015)] [article in Chinese]. Zhonghua Er Bi Yan Hou Tou Jing Wai Ke Za Zhi. 2015 Jun;50(6):443-7. 
10 Toubi E, Ben-David J, Kessel A, Halas K, Sabo E, Luntz M. Immune-mediated disorders associated with idiopathic sudden sensorineural hearing loss. Ann Otol Rhinol Laryngol. 2004 Jun;113(6):445-9.

11 Weng SF, Chen YS, Hsu CJ, Tseng FY. Clinical features of sudden sensorineural hearing loss in diabetic patients. Laryngoscope. 2005 Sep;115(9):1676-80.

12 Ciccone MM, Cortese F, Pinto M, Di Teo C, Fornarelli F, Gesualdo M, et al. Endothelial function and cardiovascular risk in patients with idiopathic sudden sensorineural hearing loss. Atherosclerosis. 2012 Dec;225(2):511-6.

13 Capaccio P, Cuccarini V, Ottaviani F, Fracchiolla NS, Bossi A, Pignataro L. Prothrombotic gene mutations in patients with sudden sensorineural hearing loss and cardiovascular thrombotic disease. Ann Otol Rhinol Laryngol. 2009 Mar;118(3):205-10.

14 Weng SF, Chen YS, Liu TC, Hsu CJ, Tseng FY. Prognostic factors of sudden sensorineural hearing loss in diabetic patients. Diabetes Care. 2004 Oct;27(10):2560-1.
15 Rudack C, Langer C, Stoll W, Rust S, Walter M. Vascular risk factors in sudden hearing loss. Thromb Haemost. 2006 Mar;95(3):45461.

16 Nacar AB, Erayman A, Kurt M, Buyukkaya E, Karakaş MF, Akcay AB, et al. The relationship between coronary collateral circulation and neutrophil/lymphocyte ratio in patients with coronary chronic total occlusion. Med Princ Pract. 2015;24(1):65-9.

17 Lattanzi S, Cagnetti C, Rinaldi C, Angelocola S, Provinciali L, Silvestrini M. Neutrophil-tolymphocyte ratio improves outcome prediction of acute intracerebral hemorrhage. J Neurol Sci. 2018 Apr;387:98-102.

18 Lattanzi S, Cagnetti C, Provinciali L, Silvestrini M. Neutrophil-to-lymphocyte ratio and neurological deterioration following acute cerebral hemorrhage. Oncotarget. 2017 Feb; 8(34):57489-94.

19 Koçak HE, Elbistanlı MS, Acıpayam H, Alakras WME, Kiral MN, Kayhan FT. Are neutrophil/lymphocyte and platelet/lymphocyte ratios related with formation of sudden hearing loss and its prognosis? Eur Ann Otorhinolaryngol Head Neck Dis. 2017 Dec;134(6):383-
20 Lee JS, Hong SK, Kim DH, Lee JH, Lee HJ, Park B, et al. The neutrophil-to-lymphocyte ratio in children with sudden sensorineural hearing loss: a retrospective study. Acta Otolaryngol. 2017 Jan;137(1):35-8.

21 Bulğurcu S, Dikilitaş B, Arslan İB, Çukurova İ. Neutrophil-to-lymphocyte and platelet-tolymphocyte ratios in pediatric patients with idiopathic sudden hearing loss. J Int Adv Otol. 2017 Aug;13(2):217-20.

22 Park YA, Kong TH, Seo YJ. A sustained increase of plasma fibrinogen in sudden sensorineural hearing loss predicts worse outcome independently. Am J Otolaryngol. 2017 JulAug;38(4):484-7.

23 Seo YJ, Jeong JH, Choi JY, Moon IS. Neutrophil-to-lymphocyte ratio and platelet-to-lymphocyte ratio: novel markers for diagnosis and prognosis in patients with idiopathic sudden sensorineural hearing loss. Dis Markers. 2014;2014:702807.

24 Kakehata S, Sasaki A, Oji K, Futai K, Ota S, Makinae K, et al. Comparison of intratympanic and intravenous dexamethasone treatment on sudden sensorineural hearing loss with diabetes. Otol Neurotol. 2006 Aug;27(5): 604-8. 